\title{
Fluctuations in the Radio Background from Intergalactic Synchrotron Emission
}

\section{Citation}

Waxman, Eli, and Abraham Loeb. 2000. "Fluctuations in the Radio Background from Intergalactic Synchrotron Emission." The Astrophysical Journal 545 (1): L11-14. https:// doi.org/10.1086/317326.

\section{Permanent link}

http://nrs.harvard.edu/urn-3:HUL.InstRepos:41393260

\section{Terms of Use}

This article was downloaded from Harvard University's DASH repository, and is made available under the terms and conditions applicable to Other Posted Material, as set forth at http:// nrs.harvard.edu/urn-3:HUL.InstRepos:dash.current.terms-of-use\#LAA

\section{Share Your Story}

The Harvard community has made this article openly available.

Please share how this access benefits you. Submit a story.

Accessibility 


\title{
FLUCTUATIONS IN THE RADIO BACKGROUND FROM INTERGALACTIC SYNCHROTRON EMISSION
}

\author{
Eli WaXman $^{1}$ And Abraham Loeb ${ }^{2}$ \\ Received 2000 July 5; accepted 2000 October 5; published 2000 November 29
}

\begin{abstract}
The shocks produced in the intergalactic medium during large-scale structure formation accelerate a population of highly relativistic electrons that emit synchrotron radiation due to intergalactic magnetic fields. In a previous paper we have shown that these electrons cool primarily by inverse Compton scattering of the microwave background photons and can thereby produce the observed intensity and spectrum of the diffuse $\gamma$-ray background. Here we calculate the intensity and angular fluctuations of the radio synchrotron background that results from the same high-energy electrons as well as the expected angular fluctuations in the $\gamma$-ray background. On angular scales smaller than a degree, the predicted fluctuations in the microwave background temperature are of order $40 \mu \mathrm{K}\left(\xi_{B} / 0.01\right)(\nu / 10 \mathrm{GHz})^{-3}$, where $\xi_{B}$ is the magnetic fraction of the postshock energy density. This foreground might have already dominated the anisotropy signal detected in existing low-frequency cosmic microwave background experiments and can be identified with confidence through multifrequency observations. Detection of the synchrotron fluctuations would allow us to determine the strength of the intergalactic magnetic field. We predict a strong correlation between high-resolution maps taken at low-frequency radio waves and at high-energy $\gamma$-rays. Young X-ray clusters may also appear as radio or $\gamma$-ray clusters. The detailed study of this correlation will become easily accessible with the future launch of the Gamma-Ray Large-Area Space Telescope.
\end{abstract}

Subject heading: diffuse radiation

\section{INTRODUCTION}

More than a third of all X-ray clusters with luminosities $\gtrsim 10^{45} \mathrm{ergs} \mathrm{s}^{-1}$ possess diffuse radio halos (Giovannini, Tordi, \& Feretti 1999). Based on energy arguments and circumstantial evidence, these radio halos are believed to be caused by synchrotron emission from shock-accelerated electrons during the merger events of their host clusters (Harris, Kapahi, \& Ekers 1980; Tribble 1993; Feretti 2000; see Liang et al. 2000 for references to alternative, less successful models). These highly relativistic electrons cool primarily through inverse Compton (IC) scattering off the cosmic microwave background (CMB). Since their cooling time is much shorter than the dynamical time of their host cluster, the radio emission is expected to last only as long as the shock persists and continues to accelerate fresh electrons to relativistic energies. Intergalactic shocks also occur along the filaments and sheets that channel mass into the clusters. These structures, also traced by the galaxy distribution (e.g., Doroshkevich et al. 1996), are induced by gravity and form as a result of converging large-scale flows in the intergalactic medium.

In a previous paper (Loeb \& Waxman 2000, hereafter LW00), we have shown that most of the diffuse $\gamma$-ray background (Sreekumar et al. 1998) might have been generated by the shocks resulting from the formation of large-scale structure in the intergalactic medium. These shocks produce a population of highly relativistic electrons with a maximum Lorentz factor $\gtrsim 10^{7}$ that scatter a small fraction of CMB photons in the present-day universe up to $\gamma$-ray energies, thereby providing the $\gamma$-ray background. The predicted flux agrees with the observed diffuse background over more than four decades in photon energy, provided that the fraction of shock energy carried by relativistic electrons is $\xi_{e} \sim 0.05$ (a value consistent with that inferred for supernovae shocks). The brightest shocks are caused by the accretion onto (or mergers of) X-ray clusters.

\footnotetext{
${ }^{1}$ Department of Condensed Matter Physics, Weizmann Institute, P.O. Box 26, Rehovot 76100, Israel; waxman@wicc.weizmann.ac.il.

${ }^{2}$ Harvard-Smithsonian Center for Astrophysics, 60 Garden Street, Cambridge, MA 02138; aloeb@cfa.harvard.edu.
}

The same electrons that emit $\gamma$-rays by IC scattering of CMB photons also produce synchrotron radiation in the radio band as a result of intergalactic magnetic fields. The existence of magnetic fields with an amplitude $\gtrsim 0.1 \mu \mathrm{G}$ is inferred in cluster halos (Kim et al. 1989; Fusco-Femiano et al. 1999; Rephaeli, Gruber, \& Blanco 1999; Kaastra et al. 1999) and is also required for the Fermi acceleration of these electrons. The appearance of radio halos around young X-ray clusters is therefore a natural consequence and an important test of our model for the extragalactic $\gamma$-ray background. The combination of radio and $\gamma$-ray data can be used to calibrate $\xi_{e}$ and determine the strength of the intergalactic magnetic field.

Intergalactic synchrotron emission contaminates CMB anisotropies and needs to be considered in the design and analysis of anisotropy experiments at low frequencies. Previous estimates of synchrotron contamination of $\mathrm{CMB}$ anisotropies focused on Galactic emission, which occurs primarily at low Galactic latitudes and large angular scales (Tegmark et al. 2000). In this Letter, we calculate the intergalactic synchrotron contribution to the fluctuations in the radio sky as a function of frequency and angular scale. We assume that most of the emission originates from the virialization shocks around X-ray clusters and use the Press-Schechter mass function to describe the abundance of such clusters as a function of redshift. Our simplified analytic calculation should be regarded only as a first estimate of the synchrotron fluctuations and could be refined with future numerical simulations (U. Keshet, E. Waxman, V. Springel, A. Loeb, \& L. Hernquist 2000, in preparation). Throughout the Letter we adopt the popular set of cosmological parameters (Ostriker \& Steinhardt 1995) with $\Omega_{\mathrm{CDM}}=0.3, \Omega_{\Lambda}=0.65, \Omega_{b}=0.05, h=0.7, \sigma_{8}=0.9$, and $n=1$. In $\S 2$ we calculate emission from single halos, and in $\S 3$ we derive the background flux and its anisotropy. The implications of our results are discussed in $\S 4$.

\section{EMISSION FROM A SINGLE HALO}

Let us first consider the luminosity produced by IC and synchrotron emission of electrons accelerated to high energies 
in the accretion shock around a halo of mass $M$ at a redshift $z$. Assuming that the shock is strong (i.e., the gas is not preheated), the population of shock-accelerated electrons acquires a number versus energy distribution with a power-law index, $p \equiv d \ln N_{e} / d \ln E=-2$ (Bell 1978; Blandford \& Ostriker 1978). The IC luminosity is then given by

$$
\nu L_{\nu}^{\mathrm{IC}}=\frac{1}{2 \ln \left(\gamma_{\max }\right)} \frac{\Omega_{b}}{\Omega_{m}} \frac{\dot{M}(M, z)}{\mu m_{p}} \xi_{e} \frac{3}{2} k T(M, z),
$$

where $\Omega_{m}=\Omega_{\mathrm{CDM}}+\Omega_{b}, \mu m_{p}$ is the average particle mass in the gas including electrons $(\mu=0.65), T$ is the postshock temperature, $\gamma_{\max } \sim 10^{7}$ is the maximum Lorentz factor of the accelerated electrons (LW00), $\dot{M}$ is the mass accretion rate across the virialization shock, and $\xi_{e}$ is the fraction of thermal shock energy carried by relativistic electrons. The synchrotron luminosity is then given by $L_{\nu}^{\mathrm{syn}} / L_{\nu}^{\mathrm{IC}}=u_{B}(M, z) / u_{\mathrm{CMB}}$, where $u_{B}$ and $u_{\mathrm{CMB}}$ are the energy densities of the postshock magnetic field and the CMB, respectively.

The velocity dispersion $\sigma$ of a halo can be related to its mass through the relation $M=(\sqrt{2} / 5) \sigma^{3}(M, z) / G H(z)$, where $H=$ $H_{0} a^{-3 / 2} g(a)$ is the Hubble parameter, with $a \equiv(1+z)^{-1}$ and $g(a)=\left[\Omega_{m}+\Omega_{\Lambda} a^{3}+\left(1-\Omega_{m}-\Omega_{\Lambda}\right) a\right]^{1 / 2}$. The numerical constant was chosen so that, for an isothermal sphere of velocity dispersion $\sigma$, the quantity $M$ is the mass enclosed within a sphere of average density $\bar{\rho}=200 \rho_{c}$, where $\rho_{c}=3 H^{2} / 8 \pi G$ is the critical density at the corresponding redshift. On dimensional grounds, the ensemble-averaged mass accretion rate onto a halo of velocity dispersion $\sigma$ can be written as (White 1994)

$$
\dot{M}(M, z)=f_{\mathrm{acc}} \frac{\sigma^{3}(M, z)}{G},
$$

while the shock temperature and radius are given by

$$
\begin{aligned}
k T(M, z) & =f_{T} \mu m_{p} \sigma^{2}(M, z), \\
r_{\mathrm{sh}}(M, z) & =f_{\mathrm{sh}} \frac{\sqrt{2}}{5} \frac{\sigma(M, z)}{H(z)} .
\end{aligned}
$$

The factors $f_{T}, f_{\text {acc }}$, and $f_{\text {sh }}$ are dimensionless numbers of order unity, which depend only weakly on the background cosmology for the relevant range of $0.3 \lesssim \Omega_{m}<1$ and should be calibrated based on numerical simulations (see, e.g., Miniati et al. 2000). We have chosen the normalization in equation (4) so that for an isothermal sphere density distribution the postshock density is $\rho_{\mathrm{sh}}=50 \rho_{c} / 3$, similar to the result for the self-similar accretion solution in an Einstein-de Sitter $\left(\Omega_{m}=1\right)$ cosmology (Bertschinger 1985). Note that in this case the mass enclosed within $r<r_{\text {sh }}$ is $2 M$.

We assume that the magnetic field energy density is a fixed fraction of the postshock energy density, $u_{B}(M, z)=$ $\xi_{B}(3 / 2) k T(M, z)\left(\Omega_{b} / \Omega_{m}\right)\left(\sigma^{2} / 2 \pi G r_{\mathrm{sh}}^{2} \mu m_{p}\right)$. Using equations (2)-(4), we find

$$
\begin{aligned}
B(M, z)= & 0.14\left(\frac{7 \Omega_{b}}{\Omega_{m}} \frac{\xi_{B}}{0.01} f_{T}\right)^{1 / 2} \\
& \times f_{\mathrm{sh}}^{-1} h_{70}^{4 / 3} a^{-2}[g(a)]^{4 / 3} M_{14}^{1 / 3} \mu \mathrm{G}
\end{aligned}
$$

where $H_{0}=70 h_{70} \mathrm{~km} \mathrm{~s}^{-1} \mathrm{Mpc}^{-1}, M=10^{14} M_{14} M_{\odot}$. Thus, for values of $\xi_{B} \sim 0.01$ well below unity, we obtain a magnetic field amplitude consistent with observations (Kim et al. 1989; Fusco-Femiano et al. 1999; Rephaeli et al. 1999; Kaastra et al. 1999) and with our model for the $\gamma$-ray background (LW00). The implied synchrotron luminosity is

$$
\begin{aligned}
\nu L_{\nu}^{\mathrm{syn}}= & 5.1 \times 10^{38} f_{\mathrm{acc}} f_{T}^{-3 / 2} f_{\mathrm{sh}}^{-2}\left(\frac{7 \Omega_{b}}{\Omega_{m}}\right)^{2} \\
& \times \frac{\xi_{B}}{0.01} \frac{\xi_{e}}{0.05} h_{70}^{2} a[g(a)]^{2} T_{\mathrm{keV}}^{7 / 2} \operatorname{ergs~s}{ }^{-1}
\end{aligned}
$$

which can be related to the cluster mass through equation (3), $M_{14}=0.54 f_{T}^{-3 / 2} h_{70}^{-1} a^{3 / 2}[g(a)]^{-1} T_{\mathrm{keV}}^{3 / 2}$, where $T_{\mathrm{keV}}=(k T / 1 \mathrm{keV})$. While $\gamma$-ray observations can be used to calibrate $\xi_{e} \sim 0.05$ (LW00), radio synchrotron observations of clusters calibrate $\xi_{B}$. The synchrotron luminosity predicted by equation (6) is consistent with the values observed for the brightest radio halos of unrelaxed X-ray clusters for $\xi_{B} \sim 0.01$ (see the upper envelope in Fig. 9 of Liang et al. 2000, obtained for $h=0.5$ and $z \sim 0.3$ ). Similar to observations, our model predicts a radio luminosity that increases with cluster temperature and a nearly flat radio spectrum (see Fig. 6 in Liang et al. 2000).

For bright clusters, the flux increment due to synchrotron emission by the nonthermal electrons accelerated in the cluster accretion shock is larger at low frequencies than the SunyaevZeldovich decrement due to the thermal intracluster electrons. For a gas density profile of an isothermal sphere,

$$
\begin{aligned}
\frac{\Delta I_{\nu}^{\mathrm{syn}}}{\Delta I_{\nu}^{\mathrm{SZ}}}= & -3.8 f_{\mathrm{acc}} f_{T} f_{\mathrm{sh}}^{-3} \frac{7 \Omega_{b}}{\Omega_{m}} \frac{\xi_{B}}{0.01} \frac{\xi_{e}}{0.05} \\
& \times h_{70}^{11 / 3} a^{5 / 2}[g(a)]^{11 / 3} M_{14}^{2 / 3} \frac{\theta d_{A}}{r_{\mathrm{sh}}}\left(\frac{\nu}{10 \mathrm{GHz}}\right)^{-3} .
\end{aligned}
$$

In the X-ray regime, the central surface brightness due to thermal bremsstrahlung emission by the intracluster gas exceeds by orders of magnitude the IC brightness; however, due to the rapid decline of the thermal brightness with projected radius outside the cluster core $\left(\propto \theta^{-3}\right)$, we find that the nonthermal brightness might dominate around the shock radius.

The above expressions apply only to clusters that possess a strong shock with a Mach number $\Upsilon \gg 1$. Nonaccreting clusters or low-mass clusters that accrete gas that was already preheated close to their virial temperature (e.g., as a result of prior collapse of their environment or because of supernova energy injection) will not show significant nonthermal emission. For moderate $\Upsilon$ shocks, accelerated electrons acquire a number-energy distribution with a power-law index $p=(r+2) /(r-1)$, where $r=8 \Upsilon^{2} /\left(6+2 \Upsilon^{2}\right)$ is the shock compression ratio for a gas with an adiabatic index $\gamma=5 / 3$. This in turn yields steep nonthermal spectra with $\nu L_{\nu}^{\mathrm{IC} \text {, syn }} \propto \nu^{-\left[2 /\left(\Upsilon^{2}-1\right)\right]}$, implying that for weak shocks with $\Upsilon \lesssim 2$, most of the nonthermal emission is at undetectable low frequencies.

\section{BACKGROUND FLUX AND ANISOTROPY}

The observed intensity at Earth of IC and synchrotron emission is given by integrating equation (1) over the halo mass distribution and cosmic time $t$. Thus,

$$
\left\langle\nu I_{\nu}^{\mathrm{IC}, \mathrm{syn}}\right\rangle=\int d z \frac{c d t}{d z} \int d M \frac{d n}{d M} \frac{\nu L_{\nu}^{\mathrm{IC}, \mathrm{syn}}(M, z)}{4 \pi(1+z)^{4}}
$$

where angular brackets denote sky average and $d n / d M$ is 
the differential number density of halos per physical volume as a function of their mass. Using the Press-Schechter mass function (Press \& Schechter 1974), we find for the cosmological parameters we have chosen $\left\langle\nu I_{\nu}^{\mathrm{IC}}\right\rangle=$ $1.5 f_{\text {acc }} f_{T}\left(\xi_{e} / 0.05\right) \mathrm{keV} \mathrm{cm} \mathrm{cm}^{-2} \mathrm{~s}^{-1} \mathrm{sr}^{-1}$. Thus, our model reproduces the $\gamma$-ray background intensity for a reasonable choice of parameters, namely, $f_{\text {acc }} f_{T}\left(\xi_{e} / 0.05\right) \approx 1$ (LW00). Given the calibration of the $\gamma$-ray background, we predict a radio background with

$$
\begin{aligned}
& \left\langle\nu I_{\nu}^{\mathrm{syn}}\right\rangle=5.0 \times 10^{-12} f_{\mathrm{acc}} \\
& \times\left(\frac{f_{T}}{f_{\mathrm{sh}}}\right)^{2} \frac{\xi_{e}}{0.05} \frac{\xi_{B}}{0.01} \text { ergs } \mathrm{cm}^{-2} \mathrm{~s}^{-1} \mathrm{sr}^{-1} .
\end{aligned}
$$

We find that $90 \%(50 \%)$ of the contribution to this background comes from massive clusters with $M>10^{14} M_{\odot}(M>5 \times$ $\left.10^{14} M_{\odot}\right)$, which are less sensitive to preheating of the accreting gas and for which the strong shock assumption is likely to apply.

The intensity fluctuations can be calculated from

$$
\begin{aligned}
\delta^{2} I(\theta) \equiv & \left\langle I_{\nu}(0) I_{\nu}(\theta)\right\rangle-\left\langle I_{\nu}\right\rangle^{2}=\int d z \frac{c d t}{d z} \int d M \\
& \times \frac{d n}{d M} \frac{\left[\nu L_{\nu}(M, z)\right]^{2}}{16 \pi^{3}(1+z)^{8} r_{\mathrm{sh}}^{2}(M, z)} P_{1,2}\left[\frac{\theta d_{A}(z)}{r_{\mathrm{sh}}(M, z)}\right] .
\end{aligned}
$$

Here $\left\langle I_{\nu}(0) I_{\nu}(\theta)\right\rangle$ is the sky average of the product of intensities along two lines of sight, 1 and 2 , separated by an angle $\theta$, $d_{A}$ is the angular diameter distance, and $P_{1,2}$ is the probability that line-of-sight 2 passes through a halo of radius $r_{\mathrm{sh}}$ given that line-of-sight 1 passes through the same halo,

$$
\begin{aligned}
P_{1,2}(x)= & \frac{2}{\pi} \int_{0}^{\pi} d \phi \int_{0}^{1} d y y \\
& \times \begin{cases}1 & \text { for }(y+x \cos \phi)^{2}+x^{2} \sin ^{2} \phi<1 \\
0 & \text { otherwise }\end{cases}
\end{aligned}
$$

We have neglected in equation (10) halo-halo correlations and assumed that the only contribution to the integral is from both lines of sight passing through the same halo. This assumption is justified since the optical depth (the average number of halos along a given line of sight) is much smaller than unity for the typical parameter values. In particular, we find that clusters with $M>5 \times 10^{14} M_{\odot}\left(M>10^{15} M_{\odot}\right)$ cover less than $10 \%(<2 \%)$ of the sky. Note that the fractional fluctuations $\delta^{2} I /\langle I\rangle^{2}$ are independent of $f_{T}, f_{\mathrm{acc}}, \xi_{e}$, and $\xi_{B}$, and their dependence on $f_{\mathrm{sh}}$ is simply given by $f_{\mathrm{sh}}^{2} \delta^{2} I\left(f_{\mathrm{sh}} \theta\right) /\langle I\rangle^{2}=\left[\delta^{2} I(\theta) /\langle I\rangle^{2}\right]_{f_{\mathrm{sh}}=1}$.

The fluctuations in the radio and $\gamma$-ray background intensities, derived from equation (10), are shown in Figure 1. Although the synchrotron background amounts to only a small fraction of the $\mathrm{CMB}$ intensity, $\left\langle I_{\nu}^{\mathrm{syn}}\right\rangle / I_{\nu}^{\mathrm{CMB}}=6 \times$ $10^{-6}\left(f_{T} / f_{\mathrm{sh}}^{2}\right)\left(\xi_{B} / 0.01\right)(\nu / 10 \mathrm{GHz})^{-3}$ for $f_{\text {acc }} f_{T}\left(\xi_{e} / 0.05\right)=1$, its fluctuations could dominate over the primordial CMB fluctuations at low photon frequencies, $\nu \lesssim 10 \mathrm{GHz}$. Our results imply that radio emission from cluster shocks contributes a fluctuation amplitude between $\sim 30$ and $50 \mu \mathrm{K}\left(f_{T} / f_{\mathrm{sh}}^{2}\right)\left(\xi_{B} / 0.01\right)(\nu / 10 \mathrm{GHz})^{-3}$ to the $\mathrm{CMB}$ on angular scales between $1^{\circ}$ and $0^{\circ} .1$, respectively. Interestingly, current anisotropy experiments are just sensitive to this level of fluctuations.

Existing detections by the Cambridge Radio Telescope

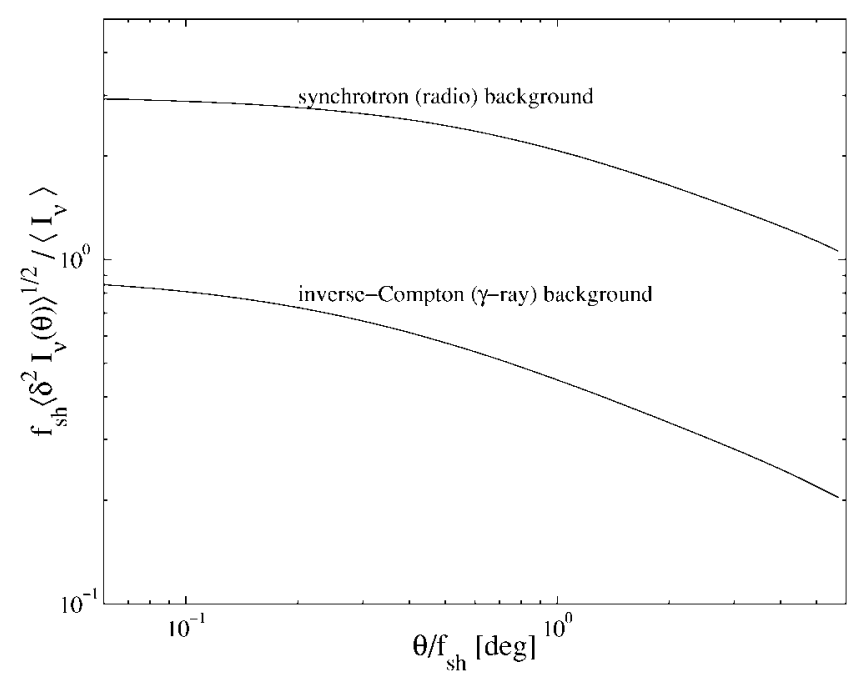

FIG. 1.-Fractional intensity fluctuations, $\left(\left\langle I_{\nu}(0) I_{\nu}(\theta)\right\rangle-\left\langle I_{\nu}\right\rangle^{2}\right)^{1 / 2} /\left\langle I_{\nu}\right\rangle$, in synchrotron (radio) and IC ( $\gamma$-ray) background flux. The dimensionless coefficient $f_{\mathrm{sh}}$ is of order unity (see definition in eq. [4]). The ratio of synchrotron to CMB intensity is $\left\langle I_{v}^{\mathrm{syn}}\right\rangle / I_{v}^{\mathrm{CMB}}=6 \times 10^{-6}\left(f_{T} / f_{\mathrm{sh}}^{2}\right)\left(\xi_{B} / 0.01\right)(\nu / 10 \mathrm{GHz})^{-3}$, where $f_{T}$ is a dimensionless coefficient of order unity (see eq. [3]), and the magnetic energy fraction $\xi_{B}$ is related to the magnetic field strength in eq. (5).

(CAT; $50 \pm 15 \mu \mathrm{K}$ at $15 \mathrm{GHz}$ on $0.2-0.5$ scales) and the Owens Valley Radio Observatory (OVRO; $56_{-6.6}^{+8.5} \mu \mathrm{K}$ at $20 \mathrm{GHz}$ on $0.1-0.6$ scales) as well as $95 \%$ upper limits $(\$ 40 \mu \mathrm{K}$ on arcminute scales at $9-15 \mathrm{GHz}$ by the Australia Telescope Compact Array, Ryle, and Very Large Array detectors) are consistent with our prediction ${ }^{3}$ (see Table 1 of White et al. 1999 for references to the experiments mentioned above).

\section{DISCUSSION}

The production of a fluctuating synchrotron background by strong intergalactic shocks is a natural consequence of structure formation in the universe. The brightest emission originates from the virialization shocks on megaparsec scales around newly formed, massive X-ray clusters. We estimate that the combined emission from all clusters produces fluctuations in the CMB temperature of order $40 \mu \mathrm{K}\left(\xi_{B} / 0.01\right)\left(\xi_{e} / 0.05\right)(\nu / 10 \mathrm{GHz})^{-3}$ on subdegree scales (Fig. 1), where $\xi_{B} \sim 0.01$ is the value required to explain the brightest radio halos of nearby clusters and $\xi_{e} \sim$ 0.05 accounts for the $\gamma$-ray background. The foreground synchrotron fluctuations might be comparable to the anisotropy signals detected by existing low-frequency CMB experiments and can be easily isolated through multifrequency observations. Polarization anisotropy experiments could then constrain the coherence length of the intergalactic magnetic field.

Our model predicts a fluctuation amplitude $\gtrsim 40 \%$ in the $\gamma$-ray background intensity on a subdegree scale (Fig. 1) and the existence of extended, $\gtrsim 1^{\circ}, \gamma$-ray halos, associated with newly formed massive clusters (Fig. 2). On scales larger than a degree the fluctuation amplitude declines and is well below the anisotropy limits from EGRET (see Fig. 5 in Sreekumar et al. 1998) although somewhat higher than expected from an analogy to the X-ray background (LW00). Detection of the predicted signals will provide a calibration of the uncertain model parameter $\xi_{e}$. The high-energy maps required to detect the predicted anisotropy

\footnotetext{
${ }^{3}$ Note that although the cluster contribution declines rapidly at high frequencies, some of these experiments, like CAT or OVRO, measured fluctuations at only one frequency and could not reject a synchrotron contribution to the measured signal.
} 

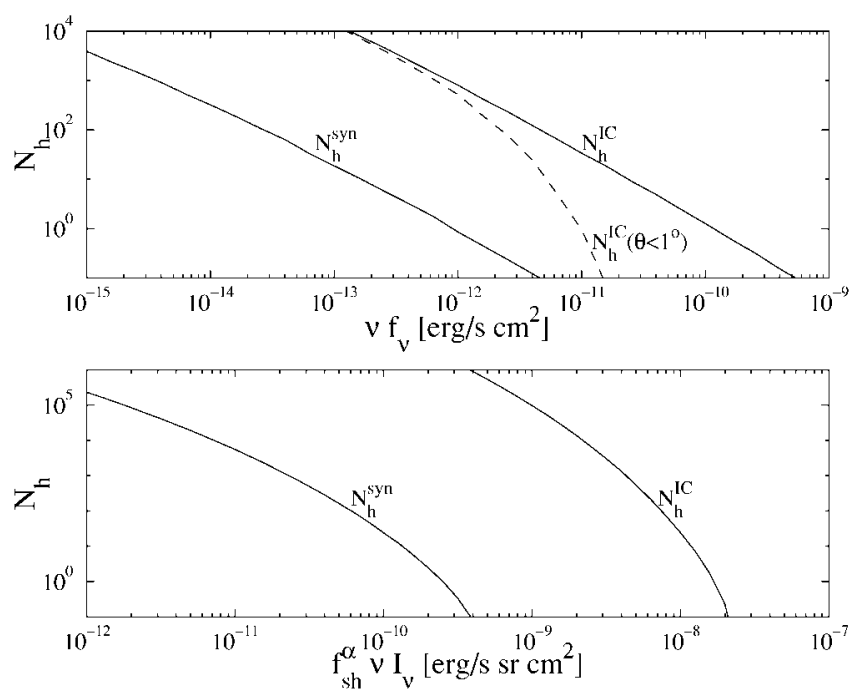

FIG. 2.-Cumulative all-sky number of IC ( $\gamma$-ray) and synchrotron (radio) halos with observed flux (top) or surface brightness (bottom) exceeding a value of $\nu f_{\nu}$ or $\nu I_{\nu}$, respectively. The normalization of the IC halo curve is fixed by the requirement that the integrated emission would produce the observed $\gamma$-ray background. The dashed line represents the number of $\gamma$-ray halos with angular radius smaller than $1^{\circ}$. The synchrotron halo flux is proportional to $\xi_{B}$, and the curve shown in the figure corresponds to $\xi_{B}=0.01$. The dimensionless coefficient $f_{\mathrm{sh}}$ is of order unity (see definition in eq. [4]); the index $\alpha=2$ for the IC emission, and $\alpha=4$ for the synchrotron emission.

signal will be made between $20 \mathrm{MeV}$ and $300 \mathrm{GeV}$ by the Gamma-Ray Large-Area Space Telescope (GLAST) ${ }^{4}$ instrument planned for launch in 2005, which is expected to be more sensitive than EGRET by an order of magnitude (Bloom 1996).

The cumulative (all sky) number of radio and $\gamma$-ray halos as a function of flux or surface brightness thresholds are shown in the top and bottom panels of Figure 2. We find that $\sim 30 \gamma$-ray halos have a flux exceeding the EGRET detection threshold of $\sim 10^{-11}$ ergs $\mathrm{cm}^{-2} \mathrm{~s}^{-1}$. Such halos may therefore constitute, as recently pointed out by Totani \& Kitayama (2000), a significant fraction of the unidentified extragalactic EGRET sources ( 60 sources over all sky; see Özel \& Thompson 1996). However, since the angular extension of the brightest halos is large, a careful analysis is required to determine their detectability by the source search analysis that was applied to EGRET data.

A future, dedicated, all-sky anisotropy experiment, operating

${ }^{4}$ See http://glast.gsfc.nasa.gov. at several frequencies below $10 \mathrm{GHz}$, would be able to map the fluctuations in the intergalactic synchrotron background. The resulting synchrotron map could then be cross-correlated with full-sky maps at hard X-ray or $\gamma$-ray energies to confirm its cosmic origin. A strong cross-correlation signal is expected since both $\gamma$-ray and radio intensity fluctuations are dominated by structures over a similar redshift range. Halos at redshifts $z \lesssim 0.1$ contribute $80 \%$ and $60 \%$ of the synchrotron (radio) and IC ( $\gamma$-ray) intensity fluctuations on an angular scale of 0.1 , and $\sim 90 \%$ of the fluctuations for both backgrounds on the 0.5 scale. Regarding the total intensity, halos at redshifts $z \lesssim 0.3$ make $80 \%$ and $60 \%$ of the synchrotron and IC backgrounds, respectively. Identification of the synchrotron fluctuations, together with their counterpart IC emission of hard X-rays or $\gamma$-rays by the same population of shock-accelerated electrons, can be used to empirically determine the strength and spatial distribution of the intergalactic magnetic fields. Similarly, the correlation between radio and $\gamma$-ray halos may be detectable around individual X-ray clusters. Strong radio halos could be the best indicators for bright $\gamma$-ray clusters, which would provide the first obvious targets for GLAST. The dominant contribution from $z<0.1$ structures to the synchrotron and IC intensity fluctuations also suggests that $\gamma$-ray and radio maps should be correlated with large-scale structure maps inferred from galaxy redshift surveys, such as the Sloan Digital Sky Survey. $^{5}$

For the sake of simplicity, our model associated the intergalactic shocks with smooth spherical accretion of gas onto clusters, while in reality they result from asymmetric mergers as well as from converging flows in large-scale sheets and filaments. The more realistic emission from these complex geometries can be best modeled through detailed hydrodynamic simulations (Miniati et al. 2000; U. Keshet, E. Waxman, V. Springel, A. Loeb, \& L. Hernquist 2000, in preparation). However, we note that mergers of comparable mass clusters would tend to produce only mild shocks due to the prior heating of the shocked gas and hence result in negligible nonthermal emission due to the steep power-law slope of the accelerated electrons.

This work was supported in part by grants from the IsraelUS BSF (BSF 98-00343) and NSF (AST 99-00877). E. W. thanks the Harvard-Smithsonian Center for Astrophysics for its kind hospitality during the course of this work. E. W. is the incumbent of the Beracha foundation career development chair.

\footnotetext{
${ }^{5}$ See http://www.sdss.org.
}

\section{REFERENCES}

Bell, A. R. 1978, MNRAS, 182, 147

Bertschinger, E. 1985, ApJS, 58, 1

Blandford, R. D., \& Ostriker, J. P. 1978, ApJ, 221, L29

Bloom, E. D. 1996, Space Sci. Rev., 75, 109

Doroshkevich, A. G., et al. 1996, MNRAS, 283, 1281

Feretti, L. 2000, in IAU Symp. 199, The Universe at Low Radio Frequencies, ed. G. Swarup \& A. Pramesh Rao (San Francisco: ASP), in press (astro$\mathrm{ph} / 0006379)$

Fusco-Femiano, R., et al. 1999, ApJ, 513, L21

Giovannini, G., Tordi, M., \& Feretti, L. 1999, NewA, 4, 141

Harris, D. E., Kapahi, V. K., \& Ekers, R. D. 1980, A\&AS, 39, 215

Kaastra, J. S., et al. 1999, ApJ, 519, L119

Kim, K.-T., Kronberg, P. P., Giovannini, G., \& Venturi, T. 1989, Nature, 341, 720

Liang, H., Hunstead, R. W., Birkinshaw, M., \& Andreani, P. 2000, ApJ, in press (astro-ph/0006072)
Loeb, A., \& Waxman, E. 2000, Nature, 405, 156 (LW00)

Miniati, F., Ryu, D., Kang, H., Jones, T. W., Cen, R., \& Ostriker, J. P. 2000, ApJ, 542, 609

Ostriker, J. P., \& Steinhardt, P. J. 1995, Nature, 377, 600

Özel, M. E., \& Thompson, D. J. 1996, ApJ, 463, 105

Press, W. H., \& Schechter, P. 1974, ApJ, 187, 425

Rephaeli, Y., Gruber, D., \& Blanco, P. 1999, ApJ, 511, L21

Sreekumar, P., et al. 1998, ApJ, 494, 523

Tegmark, M., Eisenstein, D. J., Hu, W., \& de Oliveira-Costa, A. 2000, ApJ, 530,133

Totani, T., \& Kitayama, T. 2000, ApJ, submitted (astro-ph/0006176)

Tribble, P. 1993, MNRAS, 263, 31

White, M., Carlstrom, J. E., Dragovan, M., \& Holzapfel, W. L. 1999, ApJ, 514,12

White, S. D. M. 1994, preprint (astro-ph/9410043) 